TAIWANESE JOURNAL OF MATHEMATICS

Vol. 9, No. 3, pp. 421-431, September 2005

This paper is available online at http://www.math.nthu.edu.tw/tjm/

\title{
ON THE JACOBIAN CONJECTURE
}

\author{
Lih-Chung Wang
}

\begin{abstract}
In 1983, T.T. Moh gave a computer-search algorithm to find the possible counter-example of Jacobian conjecture for polynomials of degree less than or equal to 100 . His algorithm claimed that there are only six possible counter-examples. When we re-code his algorithm, there seems to be exceptions other than his six cases. We modified Moh's algorithm with his approximate root theory to fulfill his claim.
\end{abstract}

\section{INTRODUCTION}

Let $f, g \in \mathbf{C}[x, y]$ be two polynomials with Jacobian equal to 1 . The wellknown Jacobian conjecture states " $\mathbf{C}[x, y]=\mathbf{C}[f, g]$." After the contribution of Abhyankar, Moh, Nagata et al., the possible counter-example can be written as follows:

$$
\begin{aligned}
& g=(x+y)^{p} y^{n-p}+\text { lower degree terms } \\
& f=(x+y)^{q} y^{m-q}+\text { lower degree terms }
\end{aligned}
$$

where $0<p<n-p$ and $(n-p) / p=(m-q) / q$. Note that $n-p$ can not be equal to $p$ ([2], [5]).

In 1983, Moh had established certain important properties belonging to a Jacobian pair of polynomials. These quantitative properties made a computer checking program possible and Moh succeeded in proving that the Jacobian problem is true for polynomials of degree less than or equal to 100. These subtle consequences all result from solving a sequence of differential equations discovered by Moh who effectively uses the Jacobian condition.

Our work originates from an attempt to understand Moh's algorithm. However, in re-coding his algorithm, there seems to be more exceptions than expected. In

Accepted December 26, 2003.

Communicated by Jing Yu.

2000 Mathematics Subject Classification: 14R15, 68W99.

Key words and phrases: Jacobian conjecture, Approximate root.

Paper partially supported by National Science Council (NSC-91-2115-M-259-003). 
order to make the computer checking more efficient, we modified the computation of automorphism degree and had added two checking conditions which also comes from Moh's work. This is the main result of our work. We think that the unexpected result of Moh's algorithm maybe is caused by some typos or careless writing. We hope that this paper can make the whole procedure become clear.

Section 2 aims to give a survey of Moh's work. The original definition of $A_{i}$ will be modified. In section 3, we list the exceptions and use the modified algorithm to reproduce Moh's result. Section 4 is devoted to the proof of the new criteria.

\section{SuRVEY OF MOH's THEORY}

Following S.S. Abhyankar and T.T. Moh we consider the expansion of $\{f, g\}$ at $\infty$ as follows ([5], p.149-151). Analytically, let $\mathbf{C}[x]((\eta))$ denote the ring of Laurent series with variable $\eta$ and coefficients in $\mathbf{C}[x]$. We have

$$
\begin{gathered}
g(x, y)=\eta^{-n} \\
\eta=g(x, y)^{-\frac{1}{n}}=y^{-1}+\alpha_{2}(x) y^{-2}+\cdots \in \mathbf{C}[x]\left(\left(y^{-1}\right)\right)=\mathbf{C}[x]((\eta))
\end{gathered}
$$

then

$$
f(x, y)=\eta^{-m}+\sum_{j>-m} f_{j}(x) \eta^{j} \in \mathbf{C}[x]((\eta)) .
$$

Recall [4, p.150]

$$
\begin{aligned}
d_{1} & =n, \\
M_{j} & =\min \left\{i \mid f_{i}(x) \neq 0, d_{j} \nmid i\right\}, \\
d_{j+1} & =\text { g.c.d. }\left(n, M_{1}, \cdots, M_{j}\right), \\
M_{h+1} & =\infty .
\end{aligned}
$$

The set of numbers $\left\{M_{j}, d_{j}\right\}$ are called the characteristic data of the pair $\{f, g\}$ (with respect to $x$ ).

In the above, $n-2$ must be a $M_{s}$ in the characteristic data, where either $s=h$ or $s=h-1$ ([2], [5]). We shall call $s$ the last effective index. Moh had shown that a counter-example will have $s \geq 3$.

In order to understand the fine structure of the points at infinity of the curve $g(x, y)=0$, Moh had factored $g\left(t^{-1}, y\right)$ into $\left(y-\tau_{1}\right)\left(y-\tau_{2}\right) \cdots\left(y-\tau_{n}\right)$ in the algebraically closed field $\bigcup_{k=1}^{\infty} \mathbf{C}\left(\left(t^{\frac{1}{k}}\right)\right)$. Recall that the leading form of $g(x, y)$ is $(x+y)^{p} y^{n-p}$, we can assume without loss of generality that the order of $\tau_{1}, \cdots, \tau_{n-p}$ are larger that -1 and $\tau_{n-p+1}, \cdots, \tau_{n}$ are of the form $-t^{-1}+$ higher terms in $t$. Moh had defined inductively a sequence $\{1,2, \cdots, n\} \supset I_{s} \supset I_{s-1} \supset \cdots \supset I_{2}$ by the following rules. 
- $I_{s}=\{1,2, \cdots, n-p\}$.

- Suppose that $I_{i+1}$ has been defined. Define $V_{i+1}$ and $\delta_{i}$ as follows.

$$
V_{i+1} \equiv \frac{d_{i+1}\left|I_{i+1}\right|}{n}
$$

and

$$
\delta_{i} \equiv 1-\frac{\left(n-M_{i}\right) \prod_{j=i+1}^{s}\left[V_{j}\left(n-M_{j}\right)-d_{j}\right]}{\prod_{j=i+1}^{s}\left[V_{j}\left(n-M_{j-1}\right)-d_{j}\right]} .
$$

Then $\operatorname{ord}_{t}\left(\tau_{j}-\tau_{k}\right) \geq \delta_{i}$ for all $j, k \in I_{i+1}$ and $I_{i+1}$ will be decomposed into disjoint union of subsets $I_{i+1,1}, I_{i+1,2}, \cdots$ such that $j$ and $k$ belong to the same subset if and only if $\operatorname{ord}_{t}\left(\tau_{j}-\tau_{k}\right)>\delta_{i}$.

- $I_{i+1, l}$ is called major if $\left|I_{i+1, l}\right|>\frac{n}{n-M_{i}}$, otherwise it is called minor. Choose $I_{i}$ to be any major one.

Moh's main result in [5] is that under the Jacobian condition, the sequence exists. In solving $g\left(t^{-1}, y\right)$, Moh introduced the concept of a ' $\pi$-root'

$$
\sigma=\sum_{j<\delta} a_{j} t^{j}+\pi t^{\delta}
$$

where $\sum_{j<\delta} a_{j} t^{j}$ is a partial sum of the expansion of some $\tau_{k}$. If $\sum_{j<\delta_{i}} a_{j} t^{j}$ is the common part of the expansion of $\tau_{k}$ for $k \in I_{i+1}$ and we substitute the $\pi$-root $\sigma_{i}=\sum_{j<\delta_{i}} a_{j} t^{j}+\pi t^{\delta_{i}}$ into $g\left(t^{-1}, y\right)$, we obtain

$$
g\left(t^{-1}, \sigma_{i}\right)=g_{\sigma_{i}}(\pi) t^{n \lambda_{i}}+\text { higher terms in } t .
$$

Moh proved that the order $n \lambda_{i}$ is $n \frac{-1+\delta_{i}}{n-M_{i}}$ and $g_{\sigma_{i}}(\pi)$ is of the form $p_{i}(\pi)^{\frac{n}{d_{i}}}$ with $\operatorname{deg} p_{i}(\pi)=V_{i+1} \frac{d_{i}}{d_{i+1}}$ ([5]). Denote by $c_{i}$ the coefficient of $t^{\delta_{i}}$ in the expansion of $\tau_{k}$ for $k \in I_{i}$. $\quad c_{i}$ will be a root of $p_{i}(\pi)$. However, since the $\delta_{i}$ 's are mostly fractional, the $\pi$-roots have conjugations due to the Galois extension $\mathbf{C}\left(t, c_{s-1} t^{\delta_{s-1}}, \cdots, c_{i+1} t^{\delta_{i+1}}, t^{\delta_{i}}\right)$ over $\mathbf{C}\left(t, c_{s-1} t^{\delta_{s-1}}, \cdots, c_{i+1} t^{\delta_{i+1}}\right)$. Consider the degree of the extension

$$
A_{i}=\left[\mathbf{C}\left(t, c_{s-1} t^{\delta_{s-1}}, \cdots, c_{i+1} t^{\delta_{i+1}}, t^{\delta_{i}}\right): \mathbf{C}\left(t, c_{s-1} t^{\delta_{s-1}}, \cdots, c_{i+1} t^{\delta_{i+1}}\right)\right] .
$$

When $c_{i} \neq 0, p_{i}(\pi)$ will have the factor $\left(\pi^{A_{i}}-c_{i}^{A_{i}}\right)^{V_{i}}$. If $c_{i}=0, p_{i}(\pi)$ will have the factor $\pi^{V_{i}}$ whose multiplicity $V_{i}$ is congruent to $\operatorname{deg} p_{i}(\pi)$ modulo $A_{i}$.

Note that Moh had defined $A_{i}$ to be

$$
\left[\mathbf{C}\left(t, t^{\delta_{s-1}}, \cdots, t^{\delta_{i}}\right): \mathbf{C}\left(t, t^{\delta_{s-1}}, \cdots, t^{\delta_{i+1}}\right)\right]
$$


which is different from ours. If the coefficients $c_{s-1}, \cdots, c_{i+1}$ are not zero, the two definitions are the same. Otherwise, Moh's $A_{i}$ will be a factor of ours.

Actually, Moh had considered not only $g\left(t^{-1}, y\right)$ but also $f\left(t^{-1}, y\right)$ together with a system of related polynomials (so-called approximate roots). By substituting the same $\pi$-root $\sigma_{i}$ into $f\left(t^{-1}, y\right)$, Moh can prove that $f\left(t^{-1}, \sigma_{i}\right)=f_{\sigma_{i}}(\pi) t^{m \lambda_{i}}+$ higher terms in $t=\left(p_{i}(\pi)\right)^{\frac{m}{d_{i}}} t^{m \lambda_{i}}+$ higher terms in $t$ for all $i \geq 2$. On the other hand, for $i=1, m g_{\sigma_{1}}^{\prime}(\pi) f_{\sigma_{1}}(\pi)-n g_{\sigma_{1}}(\pi) f_{\sigma_{1}}^{\prime}(\pi)$ is a nonzero constant ([5], p.187 and p.200). The coprimeness of $g_{\sigma_{1}}$ and $g_{\sigma_{1}}^{\prime}$ (resp. $f_{\sigma_{1}}$ and $f_{\sigma_{1}}^{\prime}$ ) implies that $g_{\sigma_{1}}$ (resp. $f_{\sigma_{1}}$ ) has only simple roots. Since $g_{\sigma_{1}}$ and $f_{\sigma_{1}}$ are coprime, they cannot both have $\pi$ as a factor. However, they will be polynomials in $\pi^{A_{1}}$ if both are coprime to $\pi$. This will contradict the coprimeness of $g_{\sigma_{1}}^{\prime}$ and $f_{\sigma_{1}}^{\prime}$. We conclude that one of $g_{\sigma_{1}}$ and $f_{\sigma_{1}}$ is coprime to $\pi$ and the other has $\pi$ but not $\pi^{2}$ as a factor. That is, either

or

$$
\begin{aligned}
A_{1} \mid \operatorname{deg} g_{\sigma_{1}}(\pi) & =V_{2} \frac{n}{d_{2}}, \\
A_{1} \mid\left(\operatorname{deg} f_{\sigma_{1}}(\pi)-1\right) & =V_{2} \frac{m}{d_{2}}-1
\end{aligned}
$$

$$
\begin{gathered}
A_{1} \mid V_{2} \frac{n}{d_{2}}-1, \\
A_{1} \mid V_{2} \frac{m}{d_{2}} .
\end{gathered}
$$

Therefore, any possible counter-example has to satisfy the above conditions.

\section{Moh's Algorithm}

One application of Moh's theory is to give a search algorithm of minimal counter-example for polynomials of degrees less than or equal to 100 . The searching goes as follows:

Consider a possible counter-example of minimal degree. We shall search for the sequence of integers $\left\{n, M_{1}, \cdots, M_{s}\right\}$ such that

- $\operatorname{deg} f(x, y)=m=-M_{1}<\operatorname{deg} g(x, y)=n \leq 100$,

- $m \nmid n$,

- $-m=M_{1}<M_{2}<\cdots<M_{s}=n-2$,

- $d_{r}=\operatorname{gcd}\left(n, M_{1}, \cdots, M_{r-1}\right)$ and $d_{s}<\cdots<d_{2}$.

The constraints for $s$ and $d_{s}$ are

$$
5 \geq s \geq 3
$$

and

$$
d_{s} \geq 4
$$

([5], corollary 6.1). 
Assign a sequence of integers $\left\{V_{s}, \cdots, V_{2}\right\}$ for a hypothetically existing sequence of subfamilies with

$$
V_{r+1} \frac{d_{r}}{d_{r+1}} \geq V_{r}>\frac{d_{r}}{n-M_{r}}
$$

Compute the orders $\delta_{s-1}, \cdots, \delta_{1}$ through

$$
\delta_{i}=1-\frac{\left(n-M_{i}\right) \prod_{j=i+1}^{s}\left[V_{j}\left(n-M_{j}\right)-d_{j}\right]}{\prod_{j=i+1}^{s}\left[V_{j}\left(n-M_{j-1}\right)-d_{j}\right]} .
$$

Let $L$ be the

$$
\text { l. c. m. }\left\{\text { reduced denominators of } \delta_{i}, i=s-1, \cdots, r\right\} \text {. }
$$

Denote the reduced denominator of $L \delta_{r-1}$ by $A_{r-1}$. Consider the division algorithm equation

$$
V_{r} \frac{d_{r-1}}{d_{r}}=\Delta_{r-1} A_{r-1}+\square_{r-1}
$$

The value of $V_{r-1}$ is further restricted by

$$
V_{r-1} \leq \Delta_{r-1}
$$

if the corresponding factor of $p_{r-1}(\pi)$ is of the form $(\pi-\alpha)^{V_{r-1}}$ with $\alpha \neq 0$ or

$$
A_{r-1} \mid V_{r-1}-\square_{r-1}
$$

if the corresponding factor of $p_{r-1}(\pi)$ is of the form $\pi^{V_{r-1}}$.

Finally, when $r=2$ we need to check the following conditions.

Condition 1. Either $A_{1}\left|V_{2} \frac{n}{d_{2}}, A_{1}\right| V_{2} \frac{m}{d_{2}}-1$ or $A_{1}\left|V_{2} \frac{n}{d_{2}}-1, A_{1}\right| V_{2} \frac{m}{d_{2}}$ ([5], proposition 5.5).

Condition 2. The corresponding factor of $p_{r}(\pi)$ can not be of the form $\pi^{V_{r}}$ for all $r \geq 2$ ([5], proposition 5.6).

Moh's result consists of two parts. First of all, there were six cases (four pairs of $(n, m))$ left in the computer counter-example-searching for degree $\leq 100$. They are counter-example candidates.

\begin{tabular}{|c|c|c|c|c|c|c|c|c|}
\hline$n$ & $m=-M_{1}$ & $M_{2}$ & $M_{3}$ & $M_{4}$ & $V_{3}$ & $V_{2}$ & $\delta_{2}$ & $\delta_{1}$ \\
\hline 64 & 48 & 52 & 62 & 63 & 3 & 3 & $\frac{1}{4}$ & $\frac{9}{16}$ \\
84 & 56 & $64[72]$ & 82 & 83 & 3 & $2[5]$ & $\frac{2}{7}\left[\frac{1}{4}\right]$ & $\frac{16}{21}\left[\frac{7}{12}\right]$ \\
75 & 50 & 55 & 73 & & 4 & $3[2]$ & $\frac{1}{5}$ & $\frac{1}{2}\left[\frac{2}{3}\right]$ \\
99 & 66 & 77 & 97 & & 8 & 8 & $\frac{1}{3}$ & $\frac{4}{9}$ \\
\hline
\end{tabular}


Secondly, these six candidates can further be excluded by direct computation. ([5], p.207-211)

Nevertheless, in re-coding Moh's algorithm we found that there are other cases satisfying Moh's checking criteria. For example, for $s=4$, we have

\begin{tabular}{|c|c|c|c|c|c|c|c|c|c|c|c|}
\hline$n$ & $m=-M_{1}$ & $M_{2}$ & $M_{3}$ & $M_{4}$ & $M_{5}$ & $V_{4}$ & $V_{3}$ & $V_{2}$ & $\delta_{3}$ & $\delta_{2}$ & $\delta_{1}$ \\
\hline 96 & 64 & -16 & 84 & 94 & 95 & 3 & 3 & 2 & $\frac{1}{4}$ & $\frac{9}{16}$ & $\frac{7}{12}$ \\
\hline
\end{tabular}

\begin{tabular}{|c|c|c|c|c|c|c|c|c|c|c|c|}
\hline$n$ & $m=-M_{1}$ & $M_{2}$ & $M_{3}$ & $M_{4}$ & $M_{5}$ & $V_{4}$ & $V_{3}$ & $V_{2}$ & $\delta_{3}$ & $\delta_{2}$ & $\delta_{1}$ \\
\hline 84 & 56 & 70 & 77 & 82 & 83 & 5 & 10 & 5 & $\frac{1}{4}$ & $\frac{1}{3}$ & $\frac{7}{12}$ \\
\hline
\end{tabular}

\begin{tabular}{|c|c|c|c|c|c|c|c|c|c|c|c|}
\hline$n$ & $m=-M_{1}$ & $M_{2}$ & $M_{3}$ & $M_{4}$ & $M_{5}$ & $V_{4}$ & $V_{3}$ & $V_{2}$ & $\delta_{3}$ & $\delta_{2}$ & $\delta_{1}$ \\
\hline 96 & 72 & 84 & 88 & 94 & 95 & 3 & 9 & 3 & $\frac{1}{5}$ & $\frac{1}{4}$ & $\frac{59}{80}$ \\
\hline
\end{tabular}

\begin{tabular}{|c|c|c|c|c|c|c|c|c|c|c|c|}
\hline$n$ & $m=-M_{1}$ & $M_{2}$ & $M_{3}$ & $M_{4}$ & $M_{5}$ & $V_{4}$ & $V_{3}$ & $V_{2}$ & $\delta_{3}$ & $\delta_{2}$ & $\delta_{1}$ \\
\hline 72 & 48 & 12 & 56 & 70 & 71 & 3 & 9 & 2 & $\frac{3}{11}$ & $\frac{7}{22}$ & $\frac{13}{33}$ \\
\hline
\end{tabular}

\begin{tabular}{|c|c|c|c|c|c|c|c|c|c|c|c|}
\hline$n$ & $m=-M_{1}$ & $M_{2}$ & $M_{3}$ & $M_{4}$ & $M_{5}$ & $V_{4}$ & $V_{3}$ & $V_{2}$ & $\delta_{3}$ & $\delta_{2}$ & $\delta_{1}$ \\
\hline 90 & 60 & 10 & 45 & 88 & 89 & 4 & 8 & 3 & $\frac{8}{35}$ & $\frac{5}{21}$ & $\frac{2}{7}$ \\
\hline
\end{tabular}

\begin{tabular}{|c|c|c|c|c|c|c|c|c|c|c|c|}
\hline$n$ & $m=-M_{1}$ & $M_{2}$ & $M_{3}$ & $M_{4}$ & $M_{5}$ & $V_{4}$ & $V_{3}$ & $V_{2}$ & $\delta_{3}$ & $\delta_{2}$ & $\delta_{1}$ \\
\hline 90 & 60 & 10 & 45 & 88 & 89 & 4 & 8 & 1 & $\frac{8}{35}$ & $\frac{5}{21}$ & $\frac{17}{42}$ \\
\hline
\end{tabular}

\begin{tabular}{|c|c|c|c|c|c|c|c|c|c|c|c|}
\hline$n$ & $m=-M_{1}$ & $M_{2}$ & $M_{3}$ & $M_{4}$ & $M_{5}$ & $V_{4}$ & $V_{3}$ & $V_{2}$ & $\delta_{3}$ & $\delta_{2}$ & $\delta_{1}$ \\
\hline 90 & 60 & 45 & 70 & 88 & 89 & 4 & 2 & 3 & $\frac{1}{5}$ & $\frac{2}{5}$ & $\frac{1}{2}$ \\
\hline
\end{tabular}

\begin{tabular}{|c|c|c|c|c|c|c|c|c|c|c|c|}
\hline$n$ & $m=-M_{1}$ & $M_{2}$ & $M_{3}$ & $M_{4}$ & $M_{5}$ & $V_{4}$ & $V_{3}$ & $V_{2}$ & $\delta_{3}$ & $\delta_{2}$ & $\delta_{1}$ \\
\hline 90 & 60 & 45 & 80 & 88 & 89 & 4 & 5 & 3 & $\frac{1}{7}$ & $\frac{5}{14}$ & $\frac{13}{28}$ \\
\hline
\end{tabular}

\begin{tabular}{|c|c|c|c|c|c|c|c|c|c|c|c|}
\hline$n$ & $m=-M_{1}$ & $M_{2}$ & $M_{3}$ & $M_{4}$ & $M_{5}$ & $V_{4}$ & $V_{3}$ & $V_{2}$ & $\delta_{3}$ & $\delta_{2}$ & $\delta_{1}$ \\
\hline 90 & 60 & 45 & 80 & 88 & 89 & 4 & 5 & 2 & $\frac{1}{7}$ & $\frac{5}{14}$ & $\frac{11}{21}$ \\
\hline
\end{tabular}

\begin{tabular}{|c|c|c|c|c|c|c|c|c|c|c|c|}
\hline$n$ & $m=-M_{1}$ & $M_{2}$ & $M_{3}$ & $M_{4}$ & $M_{5}$ & $V_{4}$ & $V_{3}$ & $V_{2}$ & $\delta_{3}$ & $\delta_{2}$ & $\delta_{1}$ \\
\hline 96 & 64 & 48 & 68 & 94 & 95 & 3 & 2 & 3 & $\frac{3}{10}$ & $\frac{2}{5}$ & $\frac{1}{2}$ \\
\hline
\end{tabular}

\begin{tabular}{|c|c|c|c|c|c|c|c|c|c|c|c|}
\hline$n$ & $m=-M_{1}$ & $M_{2}$ & $M_{3}$ & $M_{4}$ & $M_{5}$ & $V_{4}$ & $V_{3}$ & $V_{2}$ & $\delta_{3}$ & $\delta_{2}$ & $\delta_{1}$ \\
\hline 96 & 64 & 48 & 68 & 94 & 95 & 3 & 2 & 1 & $\frac{3}{10}$ & $\frac{2}{5}$ & $\frac{3}{4}$ \\
\hline
\end{tabular}

\begin{tabular}{|c|c|c|c|c|c|c|c|c|c|c|c|}
\hline$n$ & $m=-M_{1}$ & $M_{2}$ & $M_{3}$ & $M_{4}$ & $M_{5}$ & $V_{4}$ & $V_{3}$ & $V_{2}$ & $\delta_{3}$ & $\delta_{2}$ & $\delta_{1}$ \\
\hline 96 & 64 & 48 & 76 & 94 & 95 & 3 & 5 & 3 & $\frac{2}{7}$ & $\frac{5}{14}$ & $\frac{13}{28}$ \\
\hline
\end{tabular}

\begin{tabular}{|c|c|c|c|c|c|c|c|c|c|c|c|}
\hline$n$ & $m=-M_{1}$ & $M_{2}$ & $M_{3}$ & $M_{4}$ & $M_{5}$ & $V_{4}$ & $V_{3}$ & $V_{2}$ & $\delta_{3}$ & $\delta_{2}$ & $\delta_{1}$ \\
\hline 96 & 64 & 48 & 76 & 94 & 95 & 3 & 5 & 2 & $\frac{2}{7}$ & $\frac{5}{14}$ & $\frac{11}{21}$ \\
\hline
\end{tabular}




\begin{tabular}{|c|c|c|c|c|c|c|c|c|c|c|c|}
\hline$n$ & $m=-M_{1}$ & $M_{2}$ & $M_{3}$ & $M_{4}$ & $M_{5}$ & $V_{4}$ & $V_{3}$ & $V_{2}$ & $\delta_{3}$ & $\delta_{2}$ & $\delta_{1}$ \\
\hline 96 & 64 & 48 & 88 & 94 & 95 & 6 & 7 & 3 & $\frac{1}{5}$ & $\frac{2}{5}$ & $\frac{1}{2}$ \\
\hline
\end{tabular}

\begin{tabular}{|c|c|c|c|c|c|c|c|c|c|c|c|}
\hline$n$ & $m=-M_{1}$ & $M_{2}$ & $M_{3}$ & $M_{4}$ & $M_{5}$ & $V_{4}$ & $V_{3}$ & $V_{2}$ & $\delta_{3}$ & $\delta_{2}$ & $\delta_{1}$ \\
\hline 96 & 72 & -60 & 56 & 94 & 95 & 3 & 9 & 1 & $\frac{9}{29}$ & $\frac{19}{58}$ & $\frac{39}{116}$ \\
\hline
\end{tabular}

\begin{tabular}{|c|c|c|c|c|c|c|c|c|c|c|c|}
\hline$n$ & $m=-M_{1}$ & $M_{2}$ & $M_{3}$ & $M_{4}$ & $M_{5}$ & $V_{4}$ & $V_{3}$ & $V_{2}$ & $\delta_{3}$ & $\delta_{2}$ & $\delta_{1}$ \\
\hline 96 & 72 & 36 & 80 & 94 & 95 & 3 & 9 & 4 & $\frac{3}{11}$ & $\frac{7}{22}$ & $\frac{4}{11}$ \\
\hline
\end{tabular}

\begin{tabular}{|c|c|c|c|c|c|c|c|c|c|c|c|}
\hline$n$ & $m=-M_{1}$ & $M_{2}$ & $M_{3}$ & $M_{4}$ & $M_{5}$ & $V_{4}$ & $V_{3}$ & $V_{2}$ & $\delta_{3}$ & $\delta_{2}$ & $\delta_{1}$ \\
\hline 96 & 72 & 36 & 80 & 94 & 95 & 3 & 9 & 1 & $\frac{3}{11}$ & $\frac{7}{22}$ & $\frac{23}{44}$ \\
\hline
\end{tabular}

\begin{tabular}{|c|c|c|c|c|c|c|c|c|c|c|c|}
\hline$n$ & $m=-M_{1}$ & $M_{2}$ & $M_{3}$ & $M_{4}$ & $M_{5}$ & $V_{4}$ & $V_{3}$ & $V_{2}$ & $\delta_{3}$ & $\delta_{2}$ & $\delta_{1}$ \\
\hline 96 & 72 & 36 & 78 & 94 & 95 & 5 & 3 & 4 & $\frac{1}{7}$ & $\frac{2}{7}$ & $\frac{1}{3}$ \\
\hline
\end{tabular}

\begin{tabular}{|c|c|c|c|c|c|c|c|c|c|c|c|}
\hline$n$ & $m=-M_{1}$ & $M_{2}$ & $M_{3}$ & $M_{4}$ & $M_{5}$ & $V_{4}$ & $V_{3}$ & $V_{2}$ & $\delta_{3}$ & $\delta_{2}$ & $\delta_{1}$ \\
\hline 96 & 72 & 36 & 78 & 94 & 95 & 5 & 3 & 1 & $\frac{1}{7}$ & $\frac{2}{7}$ & $\frac{1}{2}$ \\
\hline
\end{tabular}

and, for $s=5$, we have

\begin{tabular}{|c|c|c|c|c|c|c|c|c|c|c|c|c|c|c|}
\hline$n$ & $m=-M_{1}$ & $M_{2}$ & $M_{3}$ & $M_{4}$ & $M_{5}$ & $M_{6}$ & $V_{5}$ & $V_{4}$ & $V_{3}$ & $V_{2}$ & $\delta_{4}$ & $\delta_{3}$ & $\delta_{2}$ & $\delta_{1}$ \\
\hline 96 & 64 & 80 & 88 & 92 & 94 & 95 & 3 & 5 & 10 & 5 & 0 & $\frac{1}{4}$ & $\frac{1}{3}$ & $\frac{7}{12}$ \\
\hline
\end{tabular}

\begin{tabular}{|c|c|c|c|c|c|c|c|c|c|c|c|c|c|c|}
\hline$n$ & $m=-M_{1}$ & $M_{2}$ & $M_{3}$ & $M_{4}$ & $M_{5}$ & $M_{6}$ & $V_{5}$ & $V_{4}$ & $V_{3}$ & $V_{2}$ & $\delta_{4}$ & $\delta_{3}$ & $\delta_{2}$ & $\delta_{1}$ \\
\hline 96 & 64 & -48 & -8 & 20 & 94 & 95 & 3 & 6 & 1 & 1 & $\frac{9}{28}$ & $\frac{25}{77}$ & $\frac{5}{14}$ & $\frac{3}{8}$ \\
\hline
\end{tabular}

\begin{tabular}{|c|c|c|c|c|c|c|c|c|c|c|c|c|c|c|}
\hline$n$ & $m=-M_{1}$ & $M_{2}$ & $M_{3}$ & $M_{4}$ & $M_{5}$ & $M_{6}$ & $V_{5}$ & $V_{4}$ & $V_{3}$ & $V_{2}$ & $\delta_{4}$ & $\delta_{3}$ & $\delta_{2}$ & $\delta_{1}$ \\
\hline 96 & 64 & 48 & 88 & 92 & 94 & 95 & 3 & 6 & 7 & 3 & 0 & $\frac{1}{5}$ & $\frac{2}{5}$ & $\frac{1}{2}$ \\
\hline
\end{tabular}

We modify Moh's algorithm as follows. The idea originates from Moh's work and the proof will appear in the next section (proposition 4.2,4.4).

Consider a possible counter-example of minimal degree. We shall search for the sequence of integers $\left\{n, M_{1}, \cdots, M_{s}\right\}$ such that

- $\operatorname{deg} f(x, y)=m=-M_{1}<\operatorname{deg} g(x, y)=n \leq 100$,

- $m \nmid n$,

- $-m=M_{1}<M_{2}<\cdots<M_{s}=n-2$,

- $d_{r}=\operatorname{gcd}\left(n, M_{1}, \cdots, M_{r-1}\right)$ and $d_{s}<\cdots<d_{2}$.

The constraints for $s$ and $d_{s}$ are

$$
5 \geq s \geq 3
$$


and

$$
d_{s} \geq 4
$$

Assign a sequence of integers $\left\{V_{s}, \cdots, V_{2}\right\}$ for a hypothetically existing sequence of subfamilies with

$$
V_{r+1} \frac{d_{r}}{d_{r+1}} \geq V_{r}>\frac{d_{r}}{n-M_{r}}
$$

Compute the orders $\delta_{s-1}, \cdots, \delta_{1}$ through

$$
\delta_{i}=1-\frac{\left(n-M_{i}\right) \prod_{j=i+1}^{s}\left[V_{j}\left(n-M_{j}\right)-d_{j}\right]}{\prod_{j=i+1}^{s}\left[V_{j}\left(n-M_{j-1}\right)-d_{j}\right]} .
$$

Let $L$ be the

1. c. m. $\left\{\right.$ reduced denominators of $\delta_{i}$ with $\left.V_{i} \leq \triangle_{i}, i=s-1, \cdots, r\right\}$.

Denote the reduced denominator of $L \delta_{r-1}$ by $A_{r-1}$. Consider the division algorithm equation

$$
V_{r} \frac{d_{r-1}}{d_{r}}=\Delta_{r-1} A_{r-1}+\square_{r-1}
$$

The value of $V_{r-1}$ is restricted by

$$
V_{r-1} \leq \Delta_{r-1}
$$

if the corresponding factor of $p_{r-1}(\pi)$ is of the form $(\pi-\alpha)^{V_{r-1}}$ with $\alpha \neq 0$ or

$$
A_{r-1} \mid V_{r-1}-\square_{r-1}
$$

if the corresponding factor of $p_{r-1}(\pi)$ is of the form $\pi^{V_{r-1}}$.

Finally, when $r=2$ we need to check the following conditions.

Condition 1. Either $A_{1}\left|V_{2} \frac{n}{d_{2}}, A_{1}\right| V_{2} \frac{m}{d_{2}}-1$ or $A_{1}\left|V_{2} \frac{n}{d_{2}}-1, A_{1}\right| V_{2} \frac{m}{d_{2}}$ ([5], proposition 5.5).

Condition 2. If the corresponding factor of $p_{r}(\pi)$ is of the form $\pi^{V_{r}}$ for $r=s, \cdots, i$, then $d_{2}\left(1-\delta_{i}\right) \geq n-M_{i}$ (See proposition 4.2).

Condition 3. If the corresponding factor of $p_{r}(\pi)$ is of the form $\pi^{V_{r}}$ for $r=s, \cdots, 3$, then $\square_{2}$ can not be $\frac{d_{2}}{n-M_{2}}$ (See proposition 4.4).

Furthermore, if one qualified sequence $\left\{V_{i}\right\}$ satisfies $A_{r} \nmid V_{r}-\square_{r}$ and $\square_{r}>\frac{d_{r}}{n-M_{r}}$ for some $r$, another qualified sequence $\left\{V_{s}, \cdots, V_{r+1}, V_{r}^{\prime}, \cdots\right\}$ with $A_{r} \mid V_{r}^{\prime}-\square_{r}$ 
under the same characteristic data $\left\{M_{i}, d_{i}\right\}$ should exist. Similarly, for any qualified sequence $\left\{V_{i}\right\}$ satisfying $V_{r+1} \frac{d_{r}}{d_{r+1}}>V_{r}>\triangle_{r}$ and $\frac{d_{r}}{n-M_{r}}<1$ for some $r$, another qualified sequence $\left\{V_{s}, \cdots, V_{r+1}, V_{r}^{\prime}, \cdots\right\}$ with $V_{r}^{\prime} \leq \triangle_{r}$ should exist.

With the modified algorithm, the six cases for $s=3$ are still left and all of the cases for $s=4$ or $s=5$ are ruled out.

We'd like to point out that a counter-example should reveal a whole tree of major subfamilies. Supposed that a sequence of data satisfies the checking criteria, and at the $i$-th stage, after the $V_{i}$ (resp. $I_{i}$ ) has been chosen, if the factorization of $p_{i}(\pi)$ ensures the existence of another $V_{i}$, then a second sequence of data must exist and satisfies the checking criteria also. This consideration has been included in our modified algorithm.

\section{The New Checking Criteria}

It is well known that there are a term of the form $x^{l_{n}}$ in $g(x, y)$ and a term of the form $x^{l_{m}}$ in $f(x, y)$ [3]. It is also well known that the Newton polygons of $f$ and $g$ are similar [3]. Hence, we have $\frac{n}{m}=\frac{l_{n}}{l_{m}}$ and

$$
l_{n}=\frac{n}{m} l_{m}=\frac{\frac{n}{d_{2}}}{\frac{m}{d_{2}}} l_{m} \geq \frac{n}{d_{2}}
$$

since $d_{2}$ is the g.c.d. of $m$ and $n$. Let's summarize it as the following lemma.

Lemma 4.1. There is a term of the form $x^{l_{n}}$ in $g(x, y)$ and $l_{n} \geq \frac{n}{d_{2}}$.

Proposition 2.2. Suppose that $s \geq 2$ and the $\pi$-root $\sigma_{i}$ is of the following form

$$
\sigma_{i}=\pi t^{\delta_{i}}
$$

such that

$$
d_{2}\left(1-\delta_{i}\right)<n-M_{i},
$$

then $(f, g)$ with such a $\pi$-root is not a minimal counter-example.

Proof. Suppose that $(f, g)$ is a minimal counter-example. From the above lemma, we have $g(x, y)=x^{l_{n}}+h(x, y)$ for some $h(x, y)$. Consider $g\left(t^{-1}, \sigma_{i}\right)=$ $g\left(t^{-1}, \pi t^{\delta_{i}}\right)=t^{-l_{n}}+h\left(t^{-1}, \pi t^{\delta_{i}}\right)$. Each term in $h(x, y)$ is either $x$-monomial with degree less than $l_{n}$ or a term with the $y$-variable. Therefore,

$$
n \frac{-1+\delta_{i}}{n-M_{i}}=\operatorname{ord}_{t} g\left(t^{-1}, \sigma_{i}\right) \leq-l_{n} \leq-\frac{n}{d_{2}} .
$$

i.e., 


$$
d_{2}\left(1-\delta_{i}\right) \geq n-M_{i}
$$

Corollary 4.3. ([5], proposition5.6) Suppose that $s \geq 2$ and the $\pi$-root $\sigma_{1}$ is of the following form

$$
\sigma_{1}=\pi t^{\delta_{1}}
$$

then $(f, g)$ with such a $\pi$-root is not a minimal counter-example.

Proof. We have

$$
\delta_{1}>-1
$$

Hence

$$
d_{2}\left(1-\delta_{1}\right)<2 d_{2}<n+m=n-M_{1} .
$$

Proposition 4.4. Suppose that $s \geq 3$ and that a sequence of subfamilies

$$
I_{s} \supset I_{s-1} \supset \cdots \supset I_{3}
$$

has been constructed. If the corresponding factor of $p_{r}(\pi)$ is of the form $\pi^{V_{r}}$ for $r \geq 3$ and $\square_{2}=\frac{d_{2}}{n-M_{2}}$, then $(f, g)$ with such a $\pi$-root is not a minimal counterexample.

Proof. Suppose that $(f, g)$ with such a $\pi$-root is a minimal counter-example. The multiplicity of the root zero in $p_{2}(\pi)$ is at least $\square_{2}$. If it is strictly bigger, $(f, g)$ is not a minimal counter-example by corollary 4.3. If it is $\square_{2}$, the corresponding $\pi$-root $\sigma_{1}^{*}$ is of the form

$$
\sigma_{1}^{*}=\pi t^{\delta_{1}^{*}}
$$

(corresponding to a minor case). Moh has shown that $\delta_{1}^{*} \geq 1$ ([5], proposition 6.1). It follows that

$$
\begin{aligned}
\operatorname{ord}_{t} g\left(t^{-1}, \sigma_{1}^{*}\right) & =\operatorname{ord}_{t} g\left(t^{-1}, \pi t^{\delta_{2}}\right)+V_{2} \frac{n}{d_{2}}\left(\delta_{1}^{*}-\delta_{2}\right) \\
& =n \frac{-1+\delta_{2}}{n-M_{2}}+\frac{d_{2}}{n-M 2} \frac{n}{d_{2}}\left(\delta_{1}^{*}-\delta_{2}\right) \\
& =n \frac{-1+\delta_{2}}{n-M_{2}}+n \frac{\delta_{1}^{*}-\delta_{2}}{n-M_{2}} \\
& =n \frac{-1+\delta_{1}^{*}}{n-M_{2}} \\
& \geq 0 .
\end{aligned}
$$

On the other hand, we have

$$
\operatorname{ord}_{t} g\left(t^{-1}, \sigma_{1}^{*}\right) \leq-l_{n}<0
$$

as in the proof of Proposition 4.2. This gives a contradiction. 


\section{APPENDIX}

In order to help the readers in seeing the differences between Moh's algorithm and ours, we make a comparison table.

\begin{tabular}{|l|c|c|}
\hline & Moh's algorithm & Our algorithm \\
\hline Definition of $L$ & $\operatorname{lcm}\left\{\right.$ denominators of $\left.\delta_{i}\right\}$ & $\begin{array}{c}\operatorname{lcm}\left\{\text { denominators of } \delta_{i} \text { with } V_{i} \leq \triangle_{i}\right\} \\
\text { Our } L \text { is a factor of Moh's } L .\end{array}$ \\
\hline Definition of $A_{r-1}$ & the denominator of $L \delta_{r-1}$ & $\begin{array}{c}\text { the denominator of } L \delta_{r-1} \\
\text { Our } A_{r-1} \text { is a multiple of Moh's } A_{r-1} .\end{array}$ \\
\hline Condition 2 & Corollary 4.3 & $\begin{array}{c}\text { Proposition 4.2 } \\
\text { Our condition 2 is more general. }\end{array}$ \\
\hline Condition 3 & NA & $\begin{array}{c}\text { Proposition 4.4 } \\
\text { This is a new checking condition. }\end{array}$ \\
\hline
\end{tabular}

\section{ACKNOWLEDGEMENT}

The author wishes to express his deep gratitude to Professor Moh for kindly explaining his work to him. The author also wishes to thank the referee for his suggestion.

\section{REFERENCES}

1. S. S. Abhyankar,On Expansion Techniques in Algebraic Geometry, TATA Institute, Bombay 1977.

2. H.-C. Chang and L.-C. Wang, On Group structure associated to Jacobian Pairs with maixed leading forms, J. Pure and App. Alg. 119 (1997), 1-11.

3. C. Cheng and S. Wang, Radial Similarity of Newton Polygons, Automorphisms of Affine Spaces, Kluwer Academic Publishers, (1995), 157-167.

4. R. C. Heitmann, On the Jacobian conjecture, J. Pure and App. Alg. 64 (1990), 35-72.

5. T. T. Moh, On the Jacobian conjecture and the configuration of roots, J. Reine Angew. Math. 340 (1983), 140-212.

\section{Lih-Chung Wang}

Department of Applied Mathematics,

National Donghwa University,

Shoufeng, Hualien 974,

Taiwan, R.O.C.

E-mail: lcwang@mail.ndhu.edu.tw 